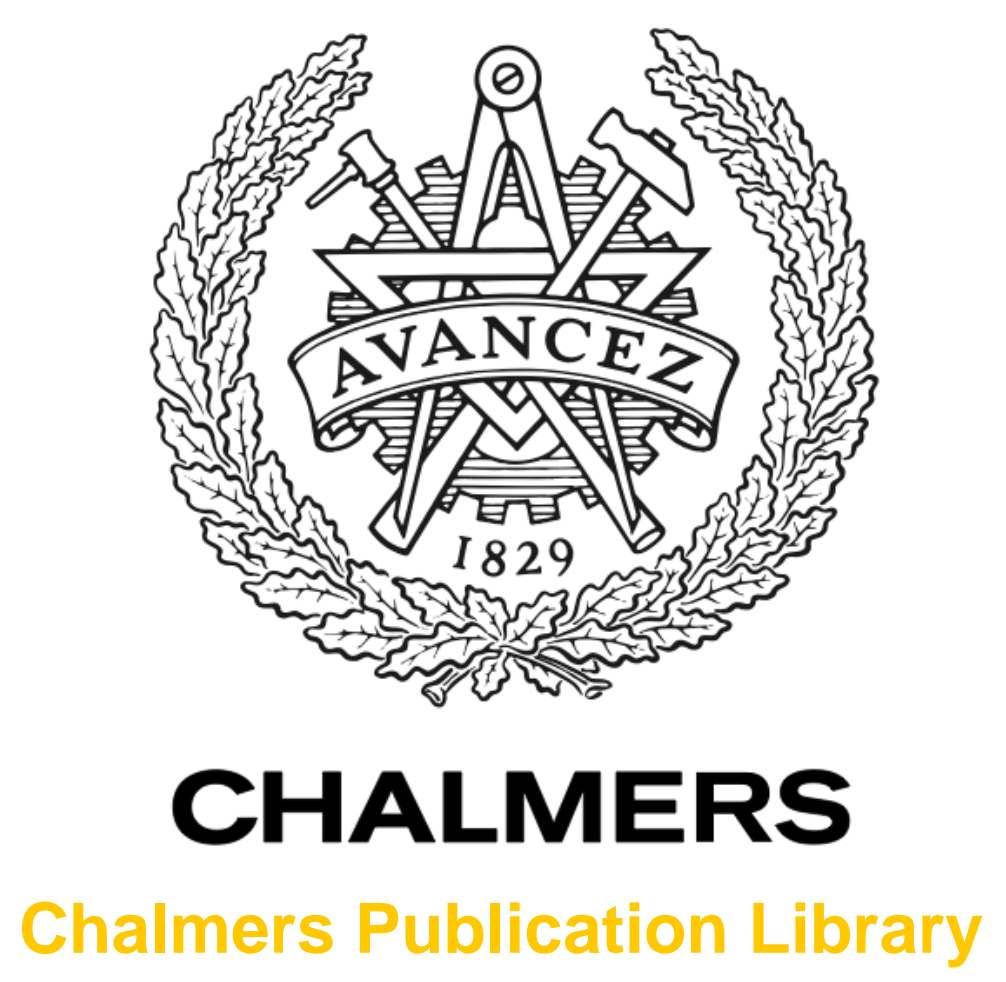

\title{
On the Throughput of the Return-Link Multi-Beam Satellite Systems using Genetic Algorithm-Based Schedulers
}

This document has been downloaded from Chalmers Publication Library $(\mathrm{CPL})$. It is the author's version of a work that was accepted for publication in:

IEEE International Conference on Communications, ICC 2015, London, United Kingdom, 812 June 2015 (ISSN: 1550-3607)

Citation for the published paper:

Makki, B. ; Svensson, T. ; Cocco, G. et al. (2015) "On the Throughput of the Return-Link Multi-Beam Satellite Systems using Genetic Algorithm-Based Schedulers". IEEE

International Conference on Communications, ICC 2015, London, United Kingdom, 8-12 June 2015 pp. 838-843.

http://dx.doi.org/10.1109/ICC.2015.7248426

Downloaded from: http://publications.lib.chalmers.se/publication/204249

Notice: Changes introduced as a result of publishing processes such as copy-editing and formatting may not be reflected in this document. For a definitive version of this work, please refer to the published source. Please note that access to the published version might require a subscription. 


\title{
On the Throughput of the Return-Link Multi-Beam Satellite Systems using Genetic Algorithm-Based Schedulers
}

\author{
Behrooz Makki*, Tommy Svensson*, Giuseppe $\mathrm{Cocco}^{\dagger}$, Tomaso de $\mathrm{Cola}^{\dagger}$, Stefan $\mathrm{Erl}^{\dagger}$ \\ *Chalmers University of Technology, Gothenburg, Sweden, \{behrooz.makki, tommy.svensson\}@ chalmers.se \\ ${ }^{\dagger}$ German Aerospace Center, DLR, 82234 Wessling, Germany, \{giuseppe.cocco,tomaso.decola,stefan.erl\}@dlr.de
}

\begin{abstract}
This paper studies the sum throughput maximization of the return-link in multi-beam satellite systems. Considering bursty communication scenarios with different users' data request probabilities, we develop an efficient scheduling scheme using genetic algorithms (GAs). Moreover, we consider co-channel interference (CCI) and adjacent channel interference (ACI). We consider a receiver with and without interference cancelation. Using a simplified channel model, we evaluate the proposed scheduler in a multi-beam system. The proposed GA-based scheduler approaches the throughput of an optimal scheduler based on exhaustive search with substantially less implementation complexity.
\end{abstract}

\section{INTRODUCTION}

The next generation of communication networks must provide data streams for everyone everywhere at any time. To address the demands, the combination of different techniques are considered among which satellite communication can play a major role [1]-[7]. Particularly, multi-beam satellite systems are efficient ways to increase the satellite capacity by re-using the available bandwidth among the beams. However, as the satellite is designed to serve a large number of users with different data request probabilities, it is essential to develop efficient scheduling schemes with affordable implementation complexity [8]-[13]. The problem becomes more important when we remember that, as discussed in e.g. [6], [10], the return-link multi-beam system is subject to co-channel interference (CCI) and adjacent channel interference (ACI) which may lead to severe performance degradation, if they are not handled appropriately.

The scheduler-based data transmission of the return-link of a multi-beam system is studied in different works. For instance, considering different types of receivers, [8] studies the bit-error-rate of the networks utilizing schedulers and interference cancellation. Implementing graph theory and successive interference cancellation, [9] introduces a low complexity heuristic algorithm for the user scheduling. Also, [10] develops a scheduling scheme based on the bipartite graph approach and [11] introduces a joint power/resource allocation method for the code division multiple access (CDMA)-based satellite return-link. Then, assuming bursty user activations, [12] proposes a scheduling algorithm for key broadcasting to minimize the number of mobile users that are prevented

978-1-4799-5863-4/14/\$31.00 (c)2014 IEEE from updating their crypto keys. Finally, minimizing the sum weighted waiting time, [13] designs a return-link scheduler by considering the data acquisition and synchronization.

In this paper we elaborate on the performance of scheduling in the return-link of a multi-beam satellite system. The problem is cast in the form of system sum-throughput optimization. Bursty communication is considered at the terminals. Resources are divided into time-frequency slots or frequencytime quanta (FTQ). At each time slot each user requests a transmission slot on a certain carrier (FTQ) with a certain probability. Both CCI and ACI are taken into account. We aim at maximizing the sum throughput of the system in different scenarios considering a receiver operating with and without successive interference cancellation. We develop an efficient scheduling scheme based on genetic algorithms (GAs). With the proposed GA-based scheduler, the appropriate data requesting users are selected and the available time-frequency units are dynamically distributed between the users such that the network sum throughput is maximized. A simplified channel model is considered and information-theoretical capacity formulas are used to evaluate the system throughput. Although, as often the case in literature, simplified assumptions are considered, the results we obtained help to get an insight in the problem, pointing out promising paths towards practical implementations. Furthermore, the algorithmic approach based on GAs is quite general, in the sense that it can adapt to different scenarios and channel models. The present work can be considered as a first step towards the development of efficient scheduling algorithms for the return link of multibeam satellite systems.

Our results indicate that 1) the proposed GA-based scheduler reaches (almost) the same performance as in the optimal exhaustive search-based scheduler, with substantially less implementation complexity. 2) The successive interference cancellation leads to substantial throughput improvements, particularly when the users' data request probability or the signal-to-noise ratio (SNR) increases. Finally, 3) the sum throughput/network diversity increases with the users' data request probability.

The problem setup of the paper is different from, e.g., [8]-[13], because we consider bursty users' data requests, the $\mathrm{CCI}$ and the ACI as well as the cases with/without 
interference cancellation. Moreover, we propose a GA-based scheduler that, up to our knowledge, has not been presented before. Finally, it is interesting to note that, while the paper concentrates on the return-link of multi-beam satellite systems, the developed scheduler could also be applied in the context of bursty multiuser multiple-input-multiple-output (MIMO) terrestrial wireless networks.

The remainder of the paper is organized as follows. In Section II, the system model is presented. The proposed GAbased scheduler is proposed in section III. In Section IV we present simulation results and investigate the effect of different parameters on the throughput. The conclusions are presented in Section V.

\section{SYSTEM MODEL}

We consider the return link of a multi beam satellite system with $b$ beams. We assume that the bandwidth is divided into sub-bands (or carriers) and time within each carrier is slotted ${ }^{1}$. We refer to a time-frequency slot as frequency-time-quantum (FTQ). For simplicity, we assume all beams to employ the same type of FTQs over time, and that in each time slot one user can be assigned to one FTQ only ${ }^{2}$.

In each FTQ of each beam, the satellite gateway receives the useful signal coming from the scheduled user together with the interfering signals. The interfering FTQs may be on the same frequency carriers on the neighbouring beams (CCI) or on neighbouring frequency carriers in the same beam (ACI).

Assume $r$ FTQs in each beam, i.e., $b r$ FTQs in total. Also, we consider a maximum of $\tilde{N} \gg b r$ users in the network, where each user may request for new data with probability $\alpha$. Also, in each time slot, the satellite is supposed to serve as many users as possible. For now, let us assume that $b r$ users are served in a time slot, while we will later study the system performance for different numbers of served users. In this way, serving $b r$ users in time slot $t$, the signal received at the gateway can be represented as

$$
\mathbf{y}(t)=\mathbf{H}(t) \mathbf{x}(t)+\mathbf{z}(t),
$$

where $\mathbf{z}(t) \in \mathcal{C N}^{b r \times 1}$ is the additive white Gaussian noise, $\mathbf{x}(t) \in \mathcal{C}^{b r \times 1}$ denotes the transmitted signal in time slot $t$ and $\mathbf{y}(t) \in \mathcal{C}^{b r \times 1}$ is the corresponding received signal. Moreover, $\mathbf{H}(t) \in \mathcal{C}^{b r \times b r}$ represents the inter-FTQ interference complex channel matrix. To simplify the presentation, in the following we drop the time index $t$. Note that the coefficients of the matrix $\mathbf{H}$ represent the FTQs in all beams and their mutual interference. Specifically, each FTQ specifies a column of $\mathbf{H}$.

To model different aspects of the channel, we define the $\mathbf{H}$ matrix as the Hadamard product of three $b r \times b r$ matrices

$$
\mathbf{H}=\mathbf{H}_{\mathrm{f}} \circ \mathbf{H}_{\mathrm{s}} \circ \mathbf{H}_{\mathrm{R}} .
$$

Here, $\circ$ denotes the Hadamard product and

\footnotetext{
${ }^{1}$ A similar approach consisting in dividing the available beam bandwidth in time-frequency allocation units is adopted in the standard second generation of Digital Video Broadcasting-Return Channel via Satellite (DVB-RCS2).

'Indeed, adaptive scaling of the users' time-frequency resources is an interesting extension of the paper.
}

- $\mathbf{H}_{\mathrm{f}}$ contains the channel coefficients which bring information on the frequency carriers, and so, on the inter-carrier interference contributions (ACI).

- $\mathbf{H}_{\mathrm{s}}$ consists of the channel coefficients which bring information on the spatial separation of the users, e.g., on the satellite beam antenna gains on the inter-beam interference (CCI).

- $\mathbf{H}_{\mathrm{R}}$ contains the channel coefficients which carries information about the atmospheric attenuation and the rain fading.

To generate $\mathbf{H}_{R}$, we consider the atmospheric attenuation and the rain fading for each user and generate the corresponding coefficient $R_{j}, j=1, \ldots, b r$. Then, $\mathbf{H}_{\mathrm{R}}$ is found as

$$
\mathbf{H}_{\mathrm{R}}=\mathbf{1}_{b r \times 1} \otimes \mathbf{R},
$$

where $\otimes$ stands for the Kronecker product, $\mathbf{R}=\left[R_{1} \ldots R_{b r}\right]$ and $\mathbf{1}_{u \times v}$ is the $u \times v$ matrix of ones.

The matrix $\mathbf{H}_{\mathrm{f}}$ is related to the ACI which exists between the carriers of a beam. In the following, we use a simplified model for the ACI, assuming that the power transfer between adjacent carriers is proportional to the interfering signal through a coefficient that is the same across beams and carriers. Formally, we define the matrix $\overline{\mathbf{H}}_{\mathrm{f}}$ of size $r \times r$ and we have

$$
\mathbf{H}_{\mathrm{f}}=\mathbf{1}_{b \times b} \otimes \overline{\mathbf{H}}_{\mathrm{f}} .
$$

As an example, setting $r=2$ we have

$$
\overline{\mathbf{H}}_{\mathrm{f}}=\left[\begin{array}{cc}
1 & h_{1,2} \\
h_{2,1} & 1
\end{array}\right] .
$$

That is, the diagonal elements of $\overline{\mathbf{H}}_{\mathrm{f}}$ are set to 1 and the offdiagonal elements represent the ACI values (for instance, $h_{2,1}$ is associated to the interference caused by carrier 1 to carrier 2).

Finally, $\mathbf{H}_{\mathrm{s}}$ contains the coefficients which are related to the users/beams and is carrier-independent. It is built starting from the matrix $\overline{\mathbf{H}}_{\mathrm{s}}$, of size $b \times b r$, which describes the channel between each user and each beam. Thus, we have

$$
\mathbf{H}_{\mathrm{s}}=\overline{\mathbf{H}}_{\mathrm{s}} \otimes \mathbf{1}_{r \times 1}
$$

and the total channel matrix is given by (2), as stated before.

In Sections III and IV, we develop the GA-based scheduling technique and evaluate the performance of the return-link multi-beam satellite systems assuming a channel matrix as the one in expression (2). Moreover, we evaluate the system performance for different users' data request probabilities and interference. Finally, note that, while we present the simulation results obtained for the channel model (2), the proposed algorithm can be applied to different channel models.

\section{Throughrut ANALYSIS}

Considering a maximum of $\tilde{N}$ users, the network sum throughput is defined as:

$$
\eta=\sum_{\forall \hat{\mathbf{H}}^{n}} \operatorname{Pr}\left(\hat{\mathbf{H}}^{n}\right) R\left(\hat{\mathbf{H}}^{n}\right) .
$$


Here, $\operatorname{Pr}\left(\hat{\mathbf{H}}^{n}\right)$ is the probability of scheduling a subset $n, \leq$ $\tilde{N}$, of the users, leading to channel matrix $\hat{\mathbf{H}}^{n}$. Note that, $\hat{\mathbf{H}}^{n}$ is a $b r \times b r$ matrix while, as seen in the following, the channel coefficients associated with non-selected FTQs are set to zero if $n<b r . R\left(\hat{\mathbf{H}}^{n}\right)$ is throughput obtained when the specific subset of users leading to channel matrix $\hat{\mathbf{H}}^{n}$ is scheduled. Moreover, the summation is on all possible channel realizations. Moreover, with the data request probability $\alpha$ for each user, we have

$$
\operatorname{Pr}\left(\hat{\mathbf{H}}^{n}\right)=\alpha^{n}(1-\alpha)^{\tilde{N}-n} .
$$

Also, the probability that, independently of the users' indices, $n$ users request for data transmission (and the rest remain silent) is given by

$$
\operatorname{Pr}(n)=\left(\begin{array}{c}
\tilde{N} \\
n
\end{array}\right) \alpha^{n}(1-\alpha)^{\tilde{N}-n}
$$

where $\left(\begin{array}{l}n \\ k\end{array}\right)$ is the binomial coefficient, which gives the number of different subsets of $k$ elements obtainable from a set of $n$.

With $b r$ FTQs, all the requesting users are scheduled if $n \leq b r$, i.e., the number of data requesting users is less than the number of available FTQs. However, to maximize the throughput, the available FTQs should be properly allocated to the users such that the impact of CCI and ACI is minimized and the network throughput is maximized. On the other hand, if $n>b r$ users request for data transmission, the scheduler selects the best br users out of $n$, such that the sum throughput is maximized. That is, with $n \leq b r$ (resp. $n>b r$ ) users the scheduler is designed for the best FTQ allocation (resp. the best user and FTQ selection).

Considering the cases with and without successive interference cancellation and for a given power per FTQ, we can use the results on the MIMO setups, e.g. [7], [14], to write ${ }^{3}$

$$
R\left(\hat{\mathbf{H}}^{n}\right)^{\mathrm{IC}}=\frac{1}{K} \log \operatorname{det}\left(\mathbf{I}_{b r}+P K \hat{\mathbf{H}}^{n}\left(\hat{\mathbf{H}}^{n}\right)^{\mathrm{h}}\right)
$$

and

$$
R\left(\hat{\mathbf{H}}^{n}\right)^{\mathrm{no}-\mathrm{IC}}=\frac{1}{K} \sum_{i=1}^{b r} \log \left(1+\frac{P \hat{g}_{i, i}^{n}}{P \sum_{j \neq i} \hat{g}_{i, j}^{n}+\frac{1}{K}}\right),
$$

respectively, where $K$ is the frequency reuse factor and $P$ denotes the transmission power normalized to the path loss (which, as the noise variance is set to 1 , represents the SNR at the receiver when $\left.g_{i, i}=1\right)$. Also, ()$^{\mathrm{h}}$ denotes the Hermitian transpose, $\mathbf{I}_{n}$ is the $n \times n$ identity channel matrix, $a_{i, j}$ is the element $(i, j)$ of the matrix $\mathbf{A}$ and we define $\hat{g}_{i, j}^{n} \doteq\left|\hat{h}_{i, j}^{n}\right|^{2}, \forall i, j$.

In this perspective, the scheduling problem is simplified to find the optimal configuration, in terms of sum throughput, of the matrix $\hat{\mathbf{H}}^{n}$ in each time slot. Using exhaustive search to find the optimal matrix configuration, the expected number of trials is of order $\mathcal{O}\left(\sum_{n=1}^{\tilde{N}} \operatorname{Pr}(n) \frac{\max (n, b r) !}{\min (n, b r) !}\right)$ which, depending

\footnotetext{
${ }^{3}$ Equations (10) and (11) represent the ultimate system performance with perfect and no interference cancellation and, thus, the throughput in the cases with imperfect interference cancellation schemes will be in between.
}

on the maximum number of users and the users' data request probability, may be quite large, making exhaustive search not practically implementable (for example, setting $\alpha=0.5, \tilde{N}=$ $100, b=20$ and $r=2$, the number of possible trials in each time slot of the exhaustive search is of the order of $2 \times 10^{80}$ ). Therefore, we need to design efficient scheduling algorithms to find the (sub)optimal resource allocation [8]-[13].

In this paper, we propose a GA-based scheduler as explained in Algorithm 1. The algorithm works as follows. Start the algorithm by selecting $M$ possible channel assignments. Each channel assignment corresponds to a selected set of users/FTQs. In each iteration, we determine the best matrix, referred to as the queen, that leads to the highest throughput, compared to the other considered matrices. Then, we keep the queen for the next iteration and create $J<M$ matrices around the queen. This is achieved by applying small modifications into the queen (for instance, by changing the FTQ assignments of two users in the queen or by replacing one user with another user in the set of users associated with the queen). Note that if $n \geq b r$ all available FTQs are utilized for data transmission and the data transmission is based on the first part of the algorithm. Otherwise, if $n \leq b r$, i.e., the number of data requesting users is less than the number of FTQs, some FTQs remain unused and their corresponding coefficients in (2)-(6) are set to zero; this case corresponds to the second part of the algorithm. Finally, in each iteration $M-J-1$ sets of users/FTQs assignments are selected randomly and the iterations continue for $N_{\text {it }}$ times considered by the designer. Running all considered iterations, the queen is returned as the scheduling rule of the current network realization. The sum throughput is calculated by averaging on the achievable rates over many channel realizations.

Considering the proposed GA-based algorithm, it is interesting to note that:

1) The algorithm is quite general, in the sense that it can adapt to different channel models and can be implemented in different network configurations.

2) As opposed to the exhaustive search-based algorithms, the proposed algorithm implies $M N_{\text {it }}$ trials of channel assignments which, depending on the considered parameter settings, can be considerably lower than in the case of exhaustive search while achieving relatively good results (see Section IV on numerical results).

3) The algorithm is presented for the buffer-limited conditions. However, it is easy to extend the algorithm to the buffer-aided scenarios where the unscheduled users are kept for the next time slots and the users are prioritized based on, e.g., their queue lengths.

4) The GA-based algorithms are commonly considered as slow optimization schemes. However, as seen in the following, the proposed algorithm leads to nearly the same results as the optimal (exhaustive-search) scheduler with a relatively small number of iterations.

5) Due to steps VII.a and V.b of the algorithm, where $M-$ $J-1$ random channel assignments are checked in each iteration, the proposed GA-based algorithm mimics the 


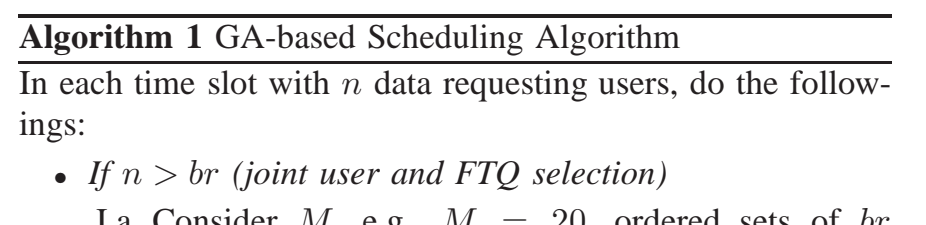

I.a Consider $M$, e.g., $M=20$, ordered sets of $b r$ users/FTQs and for each ordered set follow the same procedure as in (2)-(6) to create the channel matrix; consequently, $M$ associated matrices $\hat{\mathbf{H}}^{m, b r}, m=$ $1 \ldots, M$, are created.

II.a For each matrix $\hat{\mathbf{H}}^{m, b r}, m=1 \ldots, M$, use (10) (resp. (11)) to determine the throughput $R\left(\hat{\mathbf{H}}^{m, b r}\right)$ for the cases with interference cancellation (resp. without interference cancellation).

III.a Find the matrix which results in the highest throughput (the queen), i.e., $\hat{\mathbf{H}}^{i, b r}$ where $R\left(\hat{\mathbf{H}}^{m, b r}\right) \leq$ $R\left(\hat{\mathbf{H}}^{i, b r}\right), \forall m=1, \ldots, M$.

IV.a $\hat{\mathbf{H}}^{1, b r} \leftarrow \hat{\mathbf{H}}^{i, b r}$.

V.a Generate $J \ll M$, e.g., $J=5$, matrices $\hat{\mathbf{H}}^{j, b r, \text { new }}, j=1, \ldots, J$ around $\hat{\mathbf{H}}^{1, b r}$. These matrices are generated by small changes in the queen; for instance, by changing the order of the users in the queen (FTQ selection) or by replacing a user with another user in the ordered set of users/FTQs associated with the queen.

VI.a $\hat{\mathbf{H}}^{j+1, b r} \leftarrow \hat{\mathbf{H}}^{j, b r, \text { new }}, j=1, \ldots, J$.

VII.a Regenerate the remaining matrices $\hat{\mathbf{H}}^{j, b r}, j=J+$ $2, \ldots, M$ randomly with the same procedure as in Step I.a.

VII.a Go to II.a and continue the procedure for $N_{\text {it }}$ iterations where $N_{\text {it }}$ is the number of iterations considered by the designer.

- elseif $n \leq b r$ (FTQ selection)

I.b Consider $M$, e.g., $M=20$, ordered sets of $n$ users/FTQs to create $M$ associated matrices $\hat{\mathbf{H}}^{m, b r}, m=1 \ldots, M$. Here, the main point is that the channel coefficients associated with all nonselected FTQs are set to zero.

II.b Follow the same procedure as in Steps II.a-IV.a of the case with $n>b r$.

III.b Generate $J \ll M$, e.g., $J=5$, matrices $\hat{\mathbf{H}}^{j, b r, \text { new }}, j=1, \ldots, J$ around $\hat{\mathbf{H}}^{1, b r}$. These matrices are generated with the same procedure as in Step V.a while the derived matrices should satify the same conditions as in I.b.

IV.b $\hat{\mathbf{H}}^{j+1, b r} \leftarrow \hat{\mathbf{H}}^{j, b r, \text { new }}, j=1, \ldots, J$.

V.b Regenerate the remaining matrices $\hat{\mathbf{H}}^{j, b r}, j=J+$ $2, \ldots, M$ randomly with the same procedure as in Step I.b.

VI.b Go to II.b and continue the procedure for $N_{\text {it }}$ interations.

Return the queen as the scheduling rule of the current time slot. exhaustive search if $N_{\text {it }} \rightarrow \infty$ and it reaches the globally optimal scheduling rule if infinitely many interations are considered. That is, the proposed scheduler is optimal when the number of iterations increases asymptotically.

Further performance analysis of the developed algorithm is presented in Section IV.

\section{Simulation RESUlts}

For the simulation results, we set $K=1$. Also, we consider a maximum of $\tilde{N}=100$ users where, in different time slots, each user may request for data transmission with probability $\alpha$. Also, the results are obtained for $b=20$ beams and $r=2$ FTQs per beam, i.e., a total of 40 FTQs. With $r=2$, the matrix (4) is set to

$$
\overline{\mathbf{H}}_{\mathrm{f}}=\left[\begin{array}{ll}
1 & \beta \\
\beta & 1
\end{array}\right],
$$

where $\beta<1$ is a simulation parameter representing the mutual interference of the neighboring carriers (see (4)-(5)). Specifically, $\beta$ represents the fraction of power that is transferred from a given carrier to each of its neighbouring carriers. Also, for simplicity, we set $\mathbf{H}_{\mathrm{R}}=\mathbf{1}_{b r \times b r}$ and each element of the matrix $\overline{\mathbf{H}}_{\mathrm{s}}$ follows $\mathcal{C N}(0,1)$. The throughput is expressed in natsper-channel-use (npcu) ${ }^{4}$. Note that, along with the presented results, we have also investigated a wider range of parameters and channel conditions, but because the performances of those cases have followed the same trends as the ones shown, we have not included those results for a matter of space.

In all figures, except Fig. 2b, the algorithm is run with a sufficiently large number of iterations until no further performance improvement is observed by increasing the number of iterations. Then, in Fig. $2 b$ we investigate the performance of the proposed GA-based scheduler for different numbers of iterations.

Considering the cases with and without interference cancellation, Figs. 1a and 1b show the system throughput versus the SNR. Here, the results are obtained for different users' data request probabilities. Also, in Fig. 1a we consider different values of the interference parameter $\beta$. We observe that when implementing an ideal successive interference cancellation, the same throughput is obtained for different values of $\beta$. In Fig. $2 \mathrm{a}$ we evaluate the effect of the ACI/CCI, and show the system throughput for different values of the interference parameter $\beta$ in (12). The plots were obtained for a value of the transmit power $P$ equal to $9 \mathrm{~dB}$. The throughput shown is obtained for the case with no interference cancellation. Moreover, Fig. 2b verifies the convergence of the proposed GA-based scheduler for the cases with and without interference cancellation. Here, we set $M=20, J=5, P=9 \mathrm{~dB}, \beta=0.5, K=1$ and plot the relative achievable throughput $\Delta=\frac{\eta_{N_{\text {it }}}}{N_{\infty}} \%$, where $\eta_{N_{\text {it }}}$ is

\footnotetext{
${ }^{4}$ In the information theory, nats is the unit of information associated with the natural logarithms [15, Chapter 1]. The results of the figures can be easily mapped to the bit-per-channel-use (bpcu) scale if the y-axis of the figures are scaled by $\log _{2} e$. Moreover, the results represent the throughput in nats-persecond/hertz (npsh), if each channel use is associated with a time-frequency unit.
} 

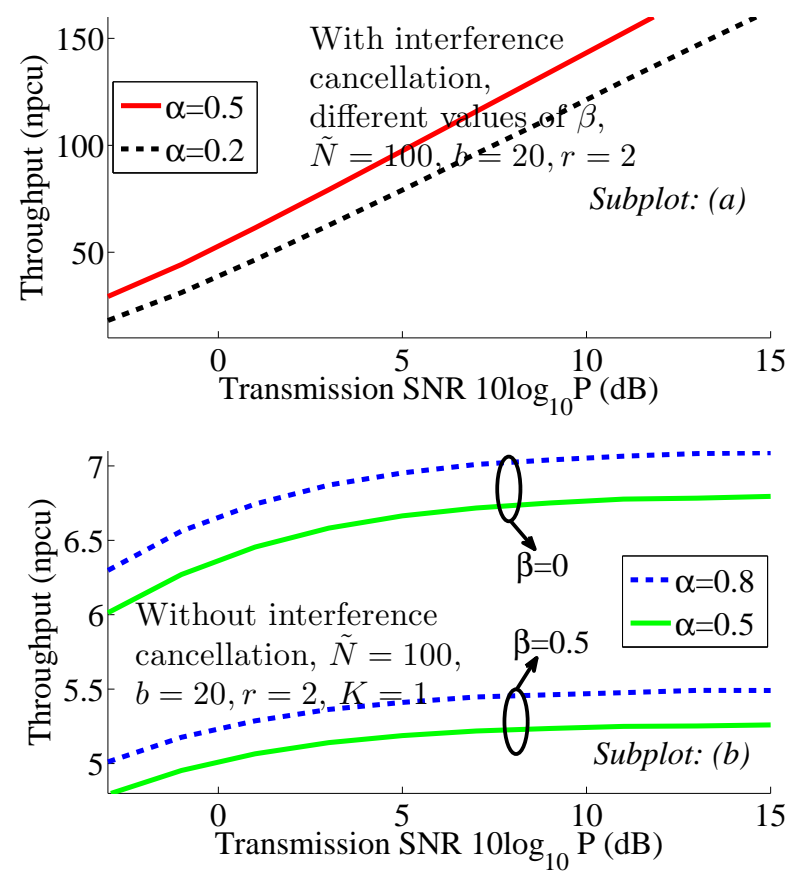

Figure 1. The network sum throughput versus the SNR $10 \log _{10} P \mathrm{~dB}, \tilde{N}=$ $100, b=20, r=2$. In subplot (a) the results are obtained with successive interference cancellation, respectively, and are independent of the interference factor $\beta$. In subplot (b) the throughput is presented for the cases without interference cancellation.

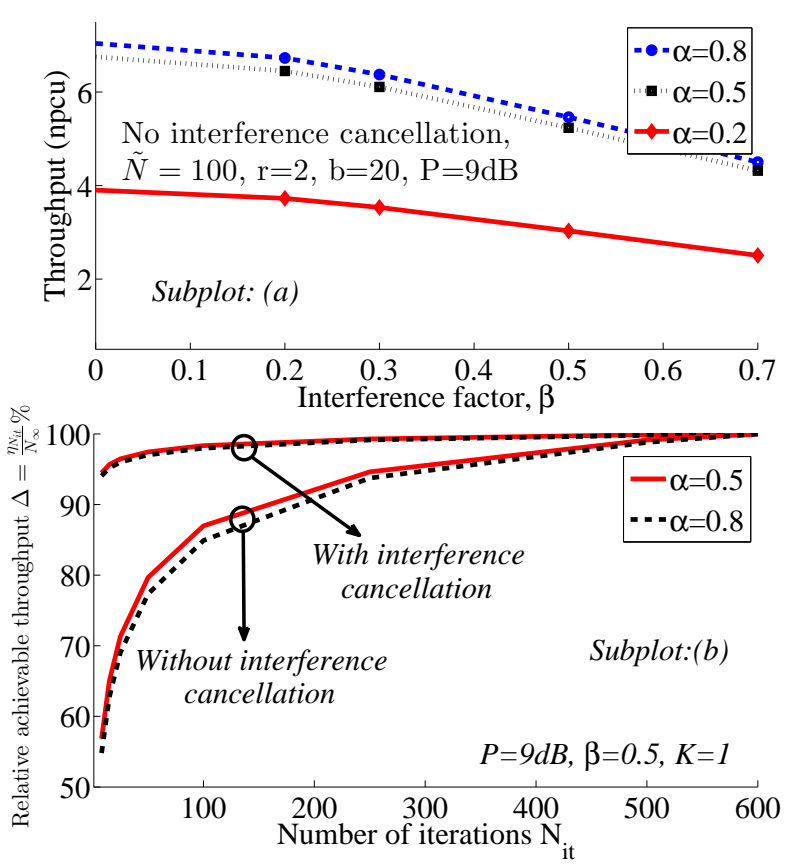

Figure 2. Subplot (a): The network sum throughput for different values of the interference parameter $\beta$, no interference cancellation, $\tilde{N}=100, b=20, r=$ 2, $P=9 \mathrm{~dB}$. Subplot (b): The relative achievable throughput $\Delta=\frac{\eta N_{\text {it }}}{N_{0}} \%$ versus the number of iterations in the proposed scheduling algorithm. The results are presented for the cases with and without interference cancellation and $M=20, K=1, \beta=0.5, P=9 \mathrm{~dB}$.

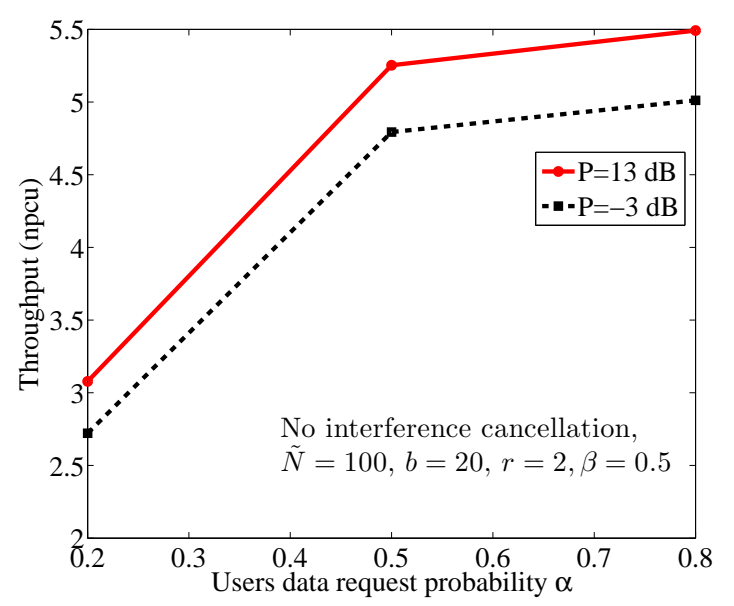

Figure 3. The network sum throughput for different values of the users' data request probability $\alpha$. The results are presented for the cases without interference cancellation and $r=2, b=20, N=100, \beta=0.5$.

the throughput achieved with $N_{\text {it }}$ number of iterations. Also, $\eta_{\infty}$ denotes the maximum achievable throughput with asymptotically high number of iterations of the algorithm, which is the same as the throughput of the exhaustive search-based scheduler. Finally, considering the cases without interference cancellation, Fig. 3 shows the system throughput for different users' data request probabilities and $\beta=0.5$.

According to the figures, we observe the following:

- On the effect of the ACI/CCI: The neighboring carriers mutual interference, modeled by the parameter $\beta$, plays an important role on the throughput, and the throughput decreases with $\beta$, if successive interference cancellation is not utilized (Figs. 1b and 2a). On the other hand, in the optimal case, the ACI is omitted and the system performance is insensitive to the parameter $\beta$ when perfect successive interference cancellation is used by the receiver (Fig. 1a). That is, with interference cancellation, the same throughput is achieved for different values of $\beta$ (Fig. 1a). Finally, the effect of the neighboring carriers mutual interference increases with the users' data request probability.

- On the performance of the proposed scheduler: As seen in Fig. 2b, the developed scheduler leads to (almost) the same performance as the exhaustive search-based scheduler with very limited number of iterations. Moreover, compared to the cases without interference cancellation, substantially better convergence rate is observed when the interference cancellation is utilized. For instance, with interference cancellation, the proposed algorithm reaches more than $95 \%$ of the maximum achievable throughput with less than 10 iterations (Fig. 2b). Also, 80\% of the maximum achievable throughput is reached with less than 70 iterations if the successive interference cancellation is not utilized; this is much less than the number of iterations required for exhaustive search. The proposed algorithm converges quite fast compared to the exhaustive search in the considered scenario. We also observe 
that the system performance improves with the number of iterations, as expected. Also, as shown in Fig. 2b, the performance of the GA-based scheduler is (almost) independent of the users' data request probability $\alpha$. The faster convergence of the algorithm in cases with interference cancellation is due to the fact that the throughput becomes independent of the ACI/CCI when the interference cancellation is used by the receiver. Therefore, the scheduling becomes easier, and the scheduler reaches the appropriate channel assignment with less number of iterations, compared to the cases without interference cancellation.

- On the effect of the interference: Without interference cancellation, the system becomes interference-limited and the sum throughput converges to constant values as the transmission power increases (Fig. 1b). On the other hand, the throughput increases with the transmission power monotonically, if successive interference cancellation is implemented (Fig. 1a). Moreover, depending on the users' data request probabilities, the interference cancellation results in substantial throughput improvements.

- On the effect of the users' bursty data request: With different interference models, the system throughput increases with the users' data request probability (Figs. $1 \mathrm{~b}, 2 \mathrm{a}$ and 3 ). Intuitively, this is because the diversity increases with more number of users requesting data and it is possible for the scheduler to select the proper users/FTQs assignments experiencing better channel qualities. However, the throughput increment, due to increasing the users' data request probabilities, is limited at high values of $\alpha$ (Fig. 3). This is because, with many users, the chance that the network becomes interferencelimited increases.

Finally, considering all figures, the interference cancellationbased scheme outperforms the case without interference cancellation, in the sense that it leads to higher throughput and requires less number of iterations in the algorithm to reach the maximum achievable throughput. However, the interference cancellation implies implementation complexities at the receiver which should be considered in practice.

\section{CONCLUSION}

This paper studied the throughput of the return-link multibeam satellite systems using schedulers. Considering different users' bursty data request probabilities, we developed a GAbased scheduler. As demonstrated, the network diversity and the throughput increase with the users' data request probability. Moreover the throughput increases substantially if successive interference cancellation is utilized at the receiver. Finally, the proposed algorithm reaches the maximum achievable throughput with relatively few iterations, which is of paramount importance from a practical implementation point of view.

\section{ACKNOWLEDGEMENT}

This research has been supported by the European Space Agency (ESA) AO7492 ARTES 5.1 3A.053 project "Efficient load-aware dynamic global radio resource management in multi-spotbeam broadband satellite network."

\section{REFERENCES}

[1] B. Bangerter, S. Talwar, R. Arefi, and K. Stewart, "Networks and devices for the 5G era," IEEE Commun. Mag., vol. 52, no. 2, pp. 90-96, Feb. 2014.

[2] J. G. Andrews, et. al, "What will 5G be?" IEEE J. Sel. Areas Commun., vol. 32, no. 6, pp. 1065-1082, June 2014.

[3] C. B. J. P. Millerioux, M. L. Boucheret and A. Ducasse, "Iterative interference cancellation and channel estimation in multibeam satellite systems," Int. J. Satell. Commun. Network., vol. 25, pp. 263-283, March 2007.

[4] M. Angelone, A. Ginesi, E. Re, and S. Cioni, "Performance of a combined dynamic rate adaptation and adaptive coding modulation technique for a DVB-RCS2 system," in ASMS, Sept. 2012, pp. 124131.

[5] N. Letzepis and A. J. Grant, "Capacity of the multiple spot beam satellite channel with Rician fading," IEEE Trans. Inf. Theory, vol. 54, no. 11, pp. 5210-5222, Nov. 2008.

[6] P. Arapoglou, K. Liolis, M. Bertinelli, A. Panagopoulos, P. Cottis, and R. De Gaudenzi, "MIMO over satellite: A review," Commun. Surveys Tuts., vol. 13, no. 1, pp. 27-51, First 2011.

[7] V. Boussemart, M. Berioli, F. Rossetto, and M. Joham, "On the achievable rates for the return-link of multi-beam satellite systems using successive interference cancellation," in MILCOM, Nov. 2011, pp. $217-$ 223

[8] V. Boussemart, L. Marini, and M. Berioli, "Multi-beam satellite MIMO systems: BER analysis of interference cancellation and scheduling," in ASMS, Sept. 2012, pp. 197-204.

[9] V. Boussemart, M. Berioli, and F. Rossetto, "User scheduling for large multi-beam satellite MIMO systems," in ASILOMAR, Nov 2011, pp. 1800-1804.

[10] V. Boussemart, L. Marini, and M. Berioli, "Impact of scheduling in the return-link of multi-beam satellite MIMO systems," in GLOBECOM, Dec. 2012, pp. 3322-3327.

[11] P. Dini, F. M. Signoretti, and R. Cusani, "Resource allocation policies for QoS guarantee service provision over a CDMA satellite return link in the aircraft broadband communication system," in ISWCS, Sept. 2005, pp. 744-748.

[12] R. Di Pietro and S. Iannitti, "Optimal key scheduling for secure satellite broadcasting to mobile users," in IWSSC, Sept. 2006, pp. 34-38.

[13] K. D. Lee, Y. H. Cho, H. J. Lee, and D. G. Oh, "Improving efficiency of timeslot assignment for non-realtime data in a DVB-RCS return link: Modeling and algorithm," ETRI Journal, vol. 25, no. 4, pp. 211-218, Aug. 2003.

[14] E. Telatar, "Capacity of multi-antenna Gaussian channels," European Trans. on Telecommun., vol. 10, pp. 585-595, 1999.

[15] T. M. Cover and J. A. Thomas, Elements of Information Theory. New York: Wiley Interscience, 1992. 